Egyptian Journal of Radiation
Sciences and Applications
http://ejra.journals.ekb.eg/

\title{
Agreements between CT, Intra-operative Inspection and Pathology Regarding Staging Local Cancer Colon
}

Eman A.S. Sabek ${ }^{\sharp}$, Gehan M. Saleh, Ehab S. Elesseily

Health Radiation Research Department, National Center of Radiation Research and Technology (NCRRT), Atomic Energy Authority (AEA), Cairo, Egypt;

\begin{abstract}
COLORECTAL carcinoma is one of the most prevalent gastrointestinal malignancy, accurate staging is very important for proper treatment. Continuous advances in CT machines, change in disease process and its pathogenicity will change the accuracy of this modality and determine weather it can be relied on specially in local staging. The aim of this study is to evaluate the accuracy of Multidetector Computed Tomography (MDCT) in local staging of colorectal cancer by comparing it to operative findings and whether surgeons can depend on its results in planning surgery, also comparing $\mathrm{CT}$ and intraoperative results to pathology reports. Hence, the strength and weakness points of CT regarding macroscopic eye inspection can be evaluated and compared to microscopic pathology findings. Regarding the T-staging, CT accurately diagnosed 83 cases, while the intraoperative examination of the specimen diagnosed only 81 cases correctly, a difference which is not statistically significant, regarding $\mathrm{N}$ stage, the intraoperative examination was more accurate than the $\mathrm{CT}$ as it correctly diagnosed 61 cases while the CT diagnosed only 56 cases correctly, a difference which is also not statistically significant. It could be concluded that CT is an accurate method in staging, especially the stages III and IV, the accuracy is still not reliable in stage I and II. Regarding $\mathrm{N}$ - staging system, CT has a low accuracy regarding pathology, this accuracy increases if compared with operative data.
\end{abstract}

Keywords: Colon cancer, Local staging. MDCT.

\section{Introduction}

Colorectal carcinoma is one of the most prevalent GIT malignancy. Knowledge of the extent of disease at initial diagnosis is very critical for proper management (Smith et al., 2007; Elias et al., 2016). Well-known important prognostic factors in colon cancer are tumor stage $(\mathrm{T})$, extramural invasion and lymph node involvement $(\mathrm{N})$. Even the total number of harvested lymph nodes at surgery and lymph node ratio (LNR) assessed by the pathologist has a prognostic importance (Chang et al., 2012; Rollvén et al., 2017). Recent developments in imaging technologies and validation of newer imaging techniques may lead to significant improvements in the management of patients with colon cancer. Multidetector Computed Tomography (MDCT) scanning, Magnetic Resonance Imaging (MRI), and Transrectal Ultrasound (TRUS) have all been extensively evaluated in initial staging of colorectal carcinoma. Although reports suggest that MRI and TRUS may provide better methods than CT for staging local colon cancer (Jhaveri \& Hosseini-Nik, 2015), up till now they have not been successful enough to be used routinely as a sole imaging modality (Jhaveri \& Hosseini-Nik, 2015)). CT scanners are now configured with multiple rows of detectors, multiplanar assessment can be performed allowing for more detailed assessment of size and morphology of pathological lesions (Anderson et al., 2011). CT is recommended in

\#Corresponding author email: hi_eman2308@yahoo.com; TEL: 0020222875924 FAX;0020222876031

Received 23/11/2019; Accepted 10/12/2019

DOI: 10.21608 /ejrsa.2019.19724.1086

C2019 National Information and Documentation Center (NIDOC) 
the initial evaluation of all patients scheduled for colorectal carcinoma surgery and for detection of its complications (obstruction, perforation, etc) (Elias et al., 2016). It is reported to be an excellent preoperative staging method with the ability to depict both tumor and metastases. The aim of this study is to evaluate the accuracy of CT scanning in staging of non-metastatic cancer in relation to operative findings. This is in addition to comparing the results of CT with those seen intraoperatively and deciding whether surgeon can depend on those results and expect the operative field and plane the treatment accordingly.

\section{Materials and Methods}

Eighty-nine patients diagnosed as having colon cancer underwent contrast enhanced CT on abdomen and pelvis, the data were collected and compared with the operative notes taken intraoperatively, and then compared with the final results of histopathology.

\section{Protocol of CT}

Toshiba 320-multidetector CT scanner machine (light speed plus; Toshiba medical system, Aqualion one, USA) was used for this study. Bowel cleansing using Picolax should be taken $24 \mathrm{~h}$ before the appointment. Picolax sachet is dissolved in a cup of cold water $(150 \mathrm{ml})$, to be well mixed for 2-3min before drinking. plenty of water must be taken until diarrhea stopped, (at least $100 \mathrm{ml} / \mathrm{h}$ ), to avoid dehydration and headaches.

Each patient receives $20 \mathrm{ml}$ of Telebrex (Meglumine ioxitalamate, Amoun, Egypt) over one and half litre of water to drink over night to opacify the intestine, last glass of water is taken immediately before the exam to opacify stomach. An intravenous line of appropriate size for IV contrast is inserted before entering the examination room. Before the examination, and on the examination table, patients are in the right lateral position, a $16 \mathrm{~F}$ tube is inserted intrarectally with administration of diluted contrast material (Ultravist 370, Iopromide, Bayer, Egypt) to the maximum tolerance of the patient. Examination starts by a scout view to determine the patient position and ensure opacification of the large and small intestine. Non intravenous contrast series are taken at this stage. Patients are injected contrast material (Ultravist 370, Iopromide, Bayer, Egypt) at a dose of $1 \mathrm{mg} / \mathrm{kg}$ by injector rate of $4 \mathrm{ml} / \mathrm{sec}$. Arterial phase starts $35 \mathrm{sec}$ after injection and is restricted to the suspected area of mass lesion. Venous phase starts after $75 \mathrm{sec}$ and includes the whole abdomen and pelvis.

\section{Interpretation}

\section{CT interpretation}

Each image is reviewed by two radiologists of at least six years of experience in abdominal imaging, who reviewed the whole series including transverse images and multiplanar reformatting MPR. Diagnosis was as follows; T1 stage was limited to the pedunculated masses with no apparent extension to the wall, T2 stage affection of the whole thickness of the wall without affection of the fat surrounding the wall, T3 stage penetration of the wall (serosa) either by nodular surface or by smudging of the fat surrounding the tumour, T4 is the affection of the whole fat plan between the mass and any adjacent organ.

Regarding N stage N1 stage is the enlargement of 3 lymph nodes or less peri-visceral in location, $\mathrm{N} 2$ is the affection of more than 3 lymph nodes peri-visceral in location, N3 is the enlargement of retroperitoneal lymph nodes, all these lymph nodes had to be $\mathrm{I} \mathrm{cm}$ in its short axis.

\section{Operative interpretation}

After excision, the mass is inspected by the surgeon and evaluated according to the staging criteria mentioned above, LN staging is also conducted, however, all lymph nodes seen in the field are excised for histopathological purpose regardless the size.

\section{Histopathological interpretation}

The staging is conducted as follows; T1 stage if there is infiltration of mucosa and sub mucosa, $\mathrm{T} 2$ infiltration of muscle layers, $\mathrm{T} 3$ penetration of the serosa by tumor cells, T4 is the affection of the adjacent organs by direct extension. Regarding $\mathrm{N}$ stage the excised nodes are examined for microscopic invasion regardless the size. For example, large nodes may be reactive in nature and small nodes can be microscopically involved by tumor cells.

\section{Statistical analysis}

Data were analysed using Statistical Package for Social Science (SPSS) version 21.0. Quantitative data were expressed as mean \pm standard deviation (SD). Qualitative data were expressed as frequency and percentage. Cohen 
Kappa was used to measure agreement between every 2 raters. Agreement is considered poor if $\mathrm{K}$ is $<0.2$, fair if between 0.21 and 0.40 , moderate if between 0.41 and 0.60 , substantial if between 0.61 and 0.80 and good if $\mathrm{K}$ is above 0.8. Sensitivity, specificity, Cohen Kappa and their confidence intervals were measured using the software Confidence Interval Analysis (CIA) for windows, developed using Borland Delphi v 4.0 (Inprise Corporation) and for help (ForeFront Technologies). P-value $<0.05$ was considered significant and P-value $<0.01$ was considered highly significant. Differences in accuracy between $\mathrm{CT}$ and operative data for $\mathrm{T}$ and $\mathrm{N}$ staging were assessed by using the McNemar test.

\section{Results}

The present study includes 89 patients diagnosed with colon cancer; most $(73 \%)$ of them were males. Age ranged from 24-82 years. Of all the diagnosed cancers, 86 cases were adenocarcinoma. Regarding the site of the lesion, rectum is the most common site diagnosed in 39 patients (43.8\%), while both ascending and descending colon tumors were the least common in our sample with only 4 (4.5\%) cases.

Regarding the $\mathrm{T}$ staging system, $\mathrm{T}_{3}$ stage was the most prevalent in in studied sample as revealed by the 3 different modalities of diagnosis; 53 cases $(59.6 \%)$ by histopathological examination., 51 cases $(57.3 \%)$ by operative inspection and 53 cases $(59.6 \%)$ by the CT. Table 1 shows the prevalence of the different $\mathrm{T}$ stages by the 3 different techniques.

Also, the 3 different diagnostic modalities agreed in that stage $\mathrm{N}_{0}$ was the most prevalent; 79 cases, 51 cases $(57.3 \%)$ by histopathological examination. $(83.1 \%)$ by intraoperative inspection, and $(88.8 \%)$ by the CT, 74 cases, the prevalence of each $\mathrm{N}$ stage by the 3 diagnostic techniques are shown in Table 2.

McNemar's test was run to determine if there is difference in the accuracy of CT and intraoperative diagnosis. Regarding the T-staging, the CT accurately diagnosed 83 cases correctly while the intraoperative examination of the specimen diagnosed only 81 cases correctly, a difference which is not statistically significant, an exact $\mathrm{P}$ value $=0.688$. However, in $\mathrm{N}$ stage, the intraoperative examination was more accurate than the CT as it correctly diagnosed 61 cases while the CT diagnosed only 56 cases correctly, a difference which is also not statistically significant, an exact $\mathrm{P}$ value of 0.063 .

TABLE 1 . Prevalence of T stage in the study.

\begin{tabular}{|c|c|c|c|}
\hline & & No. & $\%$ \\
\hline \multirow{4}{*}{ Pathology } & $\mathrm{T} 1$ & 1 & $1.1 \%$ \\
\hline & $\mathrm{T} 2$ & 26 & $29.2 \%$ \\
\hline & $\mathrm{T} 3$ & 53 & $59.6 \%$ \\
\hline & $\mathrm{T} 4$ & 9 & $10.1 \%$ \\
\hline \multirow{4}{*}{ Operative } & $\mathrm{T} 1$ & 1 & $1.1 \%$ \\
\hline & $\mathrm{T} 2$ & 25 & $28.1 \%$ \\
\hline & $\mathrm{T} 3$ & 51 & $57.3 \%$ \\
\hline & $\mathrm{T} 4$ & 12 & $13.5 \%$ \\
\hline \multirow{4}{*}{ CT } & $\mathrm{T} 1$ & 2 & $2.2 \%$ \\
\hline & $\mathrm{T} 2$ & 26 & $29.2 \%$ \\
\hline & T3 & 53 & $59.6 \%$ \\
\hline & $\mathrm{T} 4$ & 8 & $9.0 \%$ \\
\hline
\end{tabular}


TABLE 2 . Prevalence of $N$ stage in the study.

\begin{tabular}{lccc}
\hline \multirow{2}{*}{ N stage } & & No. & \% \\
\hline \multirow{3}{*}{ Pathology } & $\mathrm{N} 0$ & 51 & $57.30 \%$ \\
& $\mathrm{~N} 1$ & 23 & $25.84 \%$ \\
& $\mathrm{~N} 2$ & 13 & $14.61 \%$ \\
& $\mathrm{~N} 3$ & 2 & $2.25 \%$ \\
\hline \multirow{3}{*}{ Operative } & $\mathrm{N} 0$ & 74 & $83.14 \%$ \\
& $\mathrm{~N} 1$ & 10 & $11.24 \%$ \\
& $\mathrm{~N} 2$ & 4 & $4.50 \%$ \\
$\mathrm{CT}$ & $\mathrm{N} 3$ & 1 & $1.12 \%$ \\
\hline \multirow{3}{*}{} & $\mathrm{N} 0$ & 79 & $88.80 \%$ \\
& $\mathrm{~N} 1$ & 7 & $7.80 \%$ \\
\end{tabular}

The agreement between each two raters for the $\mathrm{T}$ and $\mathrm{N}$ stages of the TNM staging system is shown in Table 3 and is represented by the kappa coefficient together with its $95 \%$ confidence interval. The agreement is found to be good in the $\mathrm{T}$ staging between each 2 raters $(\mathrm{K}>0.8)$. However, regarding the $\mathrm{N}$ staging, it is substantial between the $\mathrm{CT}$ and the intraoperative inspection $(\mathrm{K}=0.7)$ and fair ( $\mathrm{K}$ is between 0,2 and 0.4 ) when each of the CT and the intraoperative inspection is compared to the histopathological examination.

The sensitivity, specificity, PPV and NPV of the CT in diagnosing each $\mathrm{T}$ and $\mathrm{N}$ stage are shown in Table 4, while Table 5 presents those for the intraoperative examination.

\section{Discussion}

Colorectal cancer is a common malignancy that results in significant morbidity and mortality. Surgical excision with satisfactory margins is necessary to provide a significant disease-free interval. Computed Tomography (CT) is valuable in planning surgery for colorectal cancer because it can demonstrate regional extension of tumor as well as adenopathy and distant metastases (Horton et al., 2000). Owing to the great advances in CT machines and protocols, its sensitivity and specificity reach very high levels. In the current study the sensitivity and specificity of both CT and intraoperative inspection of the specimen were measured in diagnosing each $\mathrm{T}$ and $\mathrm{N}$ stage, taking the pathological result as the gold standard test. Then a comparison was made for the overall diagnosing accuracy of both methods for the $\mathrm{T}$ and $\mathrm{N}$ staging system.
Diagnosing colorectal cancer is better done in the arterial phase because it allows better differentiation between the tumor and the adjacent organs and tissues, unlike the venous phase which results in a more homogeneous enhancement of the neoplasm and the adjacent normal tissue (Hundt et al., 1999; Kekelidze et al., 2013). Many factors have attributed to the improvement in local staging for colorectal cancer using MDCT including the rapid acquisition time, capability of using thin collimation $(4 \times 2.5 \mathrm{~mm})$ which results in improved quality of multiplanar reformatting (MPR) and better spatial resolution. Despite thin sections, an intrinsic limitation of CT is the lack of visualization of the individual wall layer (Flippone et al., 2004; Kekelidze et al., 2013)

There are few initial therapeutic options for patients with colorectal carcinoma beyond surgery. These decisions, however, cannot be made without accurate pre-surgical staging, this raises the importance of staging and continuous development of techniques used in staging of colorectal malignancies.

Initially, CT was the first "staging" modality evaluated. Early reports of accuracy that ranged between 85-90\% (Bernini et al., 1996). Flippone et al. (2004) showed that the accuracy of CT regarding $\mathrm{T}$ staging was $83 \%$ using multiplanar reformatting (MPR). Smith et al. (2007) showed much lower accuracy of $60.8 \%$ regarding T-stage. In the present study, the accuracy of CT in T-staging was over all around $93 \%$. CT diagnosed 2 cases as $\mathrm{T} 1$, one of them $(50 \%)$ proved, by pathology, to be $\mathrm{T} 2$ which means that underestimation is a high possibility in case if T1diagnosis was made by CT. This is explained by the fact that CT cannot 
accurately discriminate between different layers in the wall of the colon (Kim et al., 1999; Elias et al., 2016). Up till now reports suggest that MRI and TRUS may provide better methods than CT in differentiating layers of the colon (Kim et al., 1999; Zagoria et al., 1997). Twenty-six cases were diagnosed by CT as T2, two of them proved to be T3 by pathology $(7.7 \%)$, this may be due to microscopic invasion in pericolic fat that did not yet cause smudging or speculations as a sign of fat invasion in CT. CT diagnosed 53 cases as T3, 2 of them proved to be T2 and one shown to be T4 by pathology, the criterion that nodular surface and presence of speculation in pericolic fat means invasion was used, the 2 misinterpreted cases proved to be $\mathrm{T} 2$ that can be explained by the study of Freeny et al. (1986) who claimed that fat stranding in pericolic area can be due to fibrosis or inflammatory reaction and that the use of this criterion allows them to correctly identify 22 of 25 cases (88\%). The CT accurately diagnosed the 8 cases $(100 \%)$ of T4 stage confirmed by pathological examination, this may be due to thin collimation and decreased spacing and multiplanar capability that allow accurate judgment on the fat plans between visceral organs. This goes with larger, more carefully controlled studies which showed that the overall accuracy of $\mathrm{CT}$ is in the $50 \%-70 \%$ range, varying directly with the stage of the lesion (i.e., T4 lesions are more accurately assessed than $\mathrm{T} 2$ or $\mathrm{T} 3$ ). In the present study, it was found under staging is more common than over-staging which is consistent with So et al. ( 2017) study, unlike the study of Rotte et al. that stated that over-staging is far more common (Rotte et al., 1989). Also, it is difficult to accurately determine T-stage (depth of bowel wall penetration) on CT (Elias et al., 2016).

TABLE 3. Agreements between the three methods of diagnosis, CT, intraoperative inspection and pathology.

\begin{tabular}{lcccc}
\hline TNM staging & Agreement between & K coefficient & Standard error & 95\% Confidence interval \\
\hline \multirow{2}{*}{ T stage } & CT \& pathology & 0.878 & 0.048 & $0.783-0.972$ \\
& Pathology \& operative & 0.880 & 0.047 & $0.788-0.973$ \\
& CT \& operative & 0.881 & 0.047 & $0.788-0.973$ \\
\hline \multirow{2}{*}{ N stage } & CT \& pathology & 0.232 & 0.106 & $0.024-0.441$ \\
& Pathology \& operative & 0.355 & 0.101 & $0.157-0.553$ \\
& CT \& operative & 0.793 & 0.091 & $0.616-0.969$ \\
\hline
\end{tabular}

- Values are presented as proportions.

- Confidence intervals are presented as upper bounds, lower bounds.

TABLE 4. Sensitivity and specificity of CT for each $\mathrm{T}$ and $\mathrm{N}$ stage.

\begin{tabular}{|c|c|c|c|c|c|c|}
\hline \multirow{2}{*}{ 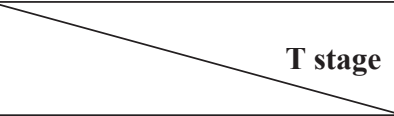 } & \multicolumn{2}{|c|}{ Stage $\leq \mathrm{T} 2$} & \multicolumn{2}{|c|}{ Stage T3 } & \multicolumn{2}{|c|}{ Stage T4 } \\
\hline & Value & $95 \%$ CI & Value & $95 \%$ CI & Value & $95 \% \mathrm{CI}$ \\
\hline Sensitivity & 0.926 & $0.766,0.979$ & 0.925 & $0.821,0.970$ & 1.000 & $0.701,1.000$ \\
\hline Specificity & 0.984 & $0.914,0.997$ & 0.944 & $0.819,0.985$ & 0.963 & $0.895,0.987$ \\
\hline Positive predictive value & 0.962 & $0.811,0.993$ & 0.961 & $0.868,0.989$ & 0.750 & $0.468,0.911$ \\
\hline \multirow[t]{3}{*}{ Negative predictive value } & 0.968 & $0.891,0.991$ & 0.895 & $0.759,0.958$ & 1.000 & $0.952,1.000$ \\
\hline & \multicolumn{2}{|c|}{ Stage No } & \multicolumn{2}{|c|}{ Stage N1 } & \multicolumn{2}{|c|}{ Stage N2 } \\
\hline & Value & $95 \%$ CI & Value & $95 \%$ CI & Value & $95 \%$ CI \\
\hline Sensitivity & 0.980 & $0.897,0.997$ & 0.261 & $0.125,0.465$ & 0.308 & $0.127,0.576$ \\
\hline Specificity & 0.368 & $0.234,0.527$ & 0.939 & $0.854,0.976$ & 1.000 & $0.952,1.000$ \\
\hline Positive predictive value & 0.676 & $0.563,0.771$ & 0.600 & $0.313,0.832$ & 1.000 & $0.510,1.000$ \\
\hline Negative predictive value & 0.933 & $0.702,0.988$ & 0.785 & $0.682,0.861$ & 0.894 & $0.811,0.943$ \\
\hline
\end{tabular}

- Values are presented as proportions.

- Confidence intervals are presented as upper bounds, lower bounds. 
TABLE 5. Sensitivity and specificity of intraoperative examination of the specimen for each $\mathbf{T}$ and $\mathbf{N}$ stage.

\begin{tabular}{|c|c|c|c|c|c|c|}
\hline & \multicolumn{2}{|c|}{ Stage $\leq \mathrm{T} 2$} & \multicolumn{2}{|c|}{ Stage T3 } & \multicolumn{2}{|c|}{ Stage T4 } \\
\hline & Value & $95 \% \mathrm{CI}$ & Value & $95 \% \mathrm{CI}$ & Value & $95 \% \mathrm{CI}$ \\
\hline Sensitivity & 0.926 & $0.766,0.979$ & 0.943 & $0.846,0.981$ & 0.889 & $0.565,0.980$ \\
\hline Specificity & 0.952 & $0.867,0.983$ & 0.917 & $0.782,0.971$ & 1.000 & $0.954,1.000$ \\
\hline Positive predictive value & 0.893 & $0.728,0.963$ & 0.943 & $0.846,0.981$ & 1.000 & $0.676,1.000$ \\
\hline \multirow[t]{3}{*}{ Negative predictive value } & 0.967 & $0.888,0.991$ & 0.917 & $0.782,0.971$ & 0.988 & $0.983,0.998$ \\
\hline & \multicolumn{2}{|c|}{ Stage No } & \multicolumn{2}{|c|}{ Stage N1 } & \multicolumn{2}{|c|}{ Stage N2 } \\
\hline & Value & $95 \% \mathrm{CI}$ & Value & $95 \% \mathrm{CI}$ & Value & $95 \% \mathrm{CI}$ \\
\hline Sensitivity & 0.980 & $0.897,0.997$ & 0.130 & $0.045,0.321$ & 0.231 & $0.082,0.503$ \\
\hline Specificity & 0.237 & $0.130,0.392$ & 0.939 & $0.854,0.976$ & 1.000 & $0.952,1.000$ \\
\hline Positive predictive value & 0.633 & $0.523,0.731$ & 0.429 & $0.158,0.750$ & 1.000 & $0.438,1.000$ \\
\hline Negative predictive value & 0.900 & $0.596,0.982$ & 0.756 & $0.653,0.836$ & 0.884 & $0.799,0.936$ \\
\hline
\end{tabular}

- Values are presented as proportions.

- Confidence intervals are presented as upper bounds, lower bounds.

The highest specificity of CT was for T4 stage, while the highest sensitivity was in diagnosing $\mathrm{T} 3$. Comparing CT staging accuracy to operative data shows a nonsignificant difference. According to these results, a surgeon can predict the operative field and accurately plane a surgery depending on $\mathrm{CT}$ results.

Looking at these results, one can observe how $\mathrm{CT}$ advances allow the accuracy to be as high as possible and be very close to the operative data. The major source of difference between CT and operative data from one side and pathology on the other side is related to microscopic type invasion. As we cannot diagnose microscopic invasion either by eye inspection or by CT scan. This kind of difference cannot be overcome preventing accuracy from reaching $100 \%$.

\section{$N$-stage}

The fact that normal sized lymph node could be microscopically invaded by tumor cells and large lymph node could be reactive cannot be denied, this may explain the lower accuracy regarding Nstaging by $\mathrm{CT}$. $\mathrm{N}$ staging accuracy in Filippone et al. was $80 \%$ in MPR (Fillppone et al., 2004). Smith et al. recorded accuracy for $\mathrm{N}$-staging was $54.3 \%$ (Smith et al., 2007), Muthusamy \& Chang (2007) showed accuracy range from 54\%-70\%. However, in the current study, CT accuracy for $\mathrm{N}$-staging was $60.86 \%$ compared to pathology results. The accuracy of CT compared to operative findings was higher reaching $83.35 \%$. Comparing the operative data with pathology, accuracy was $67.44 \%$. The accuracy is higher between CT and operative data $(83.35 \%)$ and lower between operative and pathology (67.44\%) due to the former fact of that LN can be microscopically invaded. Another factor that may aid to lower sensitivity between CT and operative data from one side and the pathology on the other side is that there is a little agreement on the critical cut-off diameter to determine if lymph nodes are involved in the disease process. The more accepted one is bigger than or equal to $1 \mathrm{~cm}$ (Fillppone et al., 2004; Elias et al., 2016), other studies suggest $5 \mathrm{~mm}$ (Ju et al., 2009; Dighe et al., 2010). However, nodal size is not seen as a predictor of nodal status at surgery (Muthusamy \& Chang, 2007; Low et al., 2008; Ong \& Schofield, 2016; So et al., 2017). Since detection of nodes involved with tumor remains a difficult problem, if a colonic resection is planned, local node groups are encompassed in a properly performed cancer operation. This leads to overcoming the lower accuracy of the CT in detecting LN invasion (Gerard et al., 1996; Thoeni, 1997; Sigurdson, 2003; Okada et al., 2015).

\section{Conclusions}

MDCT is a reliable method for staging local colorectal cancer regarding stage III and IV, however, early stages of colorectal cancer still cannot be accurately detected because MDCT cannot differentiate between different layers of the colon. Regarding N staging, it is of a lower accuracy that can be overcome during surgery by removing all LN seen in the field. However, comparing operative data and $\mathrm{CT}$ results 
the difference is insignificant. Surgeons can accurately expect what they will see depending on CT results, accordingly they can plan their surgery in a reliable manner.

Conflict of interest: The authors have nothing to disclose.

\section{$\underline{\text { References }}$}

Anderson, E.M., Betts, M., Slater, A. (2011) The value of true axial imaging for CT staging of colonic cancer. Eur Radiol. 21, 1286-92.

Bernini, A., Deen, K.I., Madoff, R.D., Wong, W.D. (1996) Preoperative adjuvant radiation with chemotherapy for rectal cancer: Its impact on stage of disease and the role of endorectal ultrasound. Ann. Surg. Onco. 3(2), 131-135.

Chang, Y.J., Chang, Y.J., Chen, L.J., Chung, K.P., Lai M.S. (2012) Evaluation of lymph nodes in patients with colon cancer undergoing colon resection: A population-based study. World J. Surg. 36, 190614.

Dighe, S., Blake, H., Koh, M.D. (2010) Accuracy of multidetector computed tomography in identifying poor prognostic factors in colonic cancer. $\mathrm{Br}$. J. Surg. 97, 1407-141.

Elias, N.M., Lahaye, J., Maas, M., Nelemans, P., Bakers F.C.H., Beets, G.L., Beets-Tan, Regina G.H. (2016) Diagnostic accuracy of CT for local staging of colon cancer: A systematic review and meta-analysis. American Journal of Roentgenology, 207(5), 984-995.

Farouk, R., Nelson, H., Radice, E., Mercill, S., Gunderson, L. (1998) Accuracy of computed tomography in determining resectability for locally advanced primary or recurrent colorectal cancers. Am. J. Surg. 175(4), 283-287.

Flippone, A., Ambrosini, R., Fuschi, M., Marinelli, T., Genovesi, D., Bonomo, L. (2004) Preoperative $\mathrm{T}$ and $\mathrm{M}$ staging of colorectal cancer: Accuracy of contrast -enhanced multi-detector row CT colonography-initial experience. Radiology, 231, 83-90.

Freeny, P.C., Marks, W.M., Ryan, J.A., et al. (1986) Colorectal carcinoma evaluation with CT: preoperative staging and detection of postoperative recurrence. Radiology, 158, 347-353.

Gerard, J.P., Ayzac, L., Coquard, R., et al (1996) Endocavitary irradiation for early rectal carcinomas T1 (T2). A series of 101 patients treated with the Papillon's technique. Int. J. Radiat. Oncol. Biol. Phys. 34(4), 775-783.

Horton, K.M., Abrams, R.A., Fishman, E.K. (2000) Spiral CT of colon cancer: Imaging features and role in management. Radio Graphics, 20, 419-430.

Hundt, W., Braunschweig, R., Reiser, M. (1999) Evaluation of spiral CT in staging of colon and rectum carcinoma. Eur. Radiol, 9, 78-84.

Ju, H., Xu, D., Li, D., Chen, G., Shao, G. (2009) Comparison between endoluminal ultrasonography and spiral computerized tomography for the preoperative local staging of rectal carcinoma. Biosci Trends, 3(2), 73-76.

Jhaveri, K.S., Hosseini-Nik, H. (2015) MRI of rectal cancer: An overview and update on recent advances. American Journal of Roentgenology, 205(1), 42-55.

Kekelidze, M., D’Errico, L., Pansini, M., Tyndall, A., Hohmann, J. (2013) Colorectal cancer: Current imaging methods and future perspectives for the diagnosis, staging and therapeutic response evaluation. World Journal of Gastroenterology, 19(46), 8502-8514.

Kim, N.K., Kim, M.J., Yun, S.H., Sohn, S.K., Min, J.S. (1999) Comparative study of transrectal ultrasonography, pelvic computerized tomography, and magnetic resonance imaging in preoperative staging of rectal cancer. Dis Colon Rectum, 42, 770-775.

Low, G., Tho, L.M., Leen, E., et al., (2008) The role of imaging in the pre-operative staging and postoperative follow-up of rectal cancer. Surgeon, 6(4), 222-231.

Muthusamy, V.R., Chang, K.J. (2007) Optimal methods for staging rectal cancer. Clin. Cancer Res. November, 13(22), 6877-6884.

Okada, K., Sadahiro, S., Suzuki, T., Tanaka, A., Saito, G., Masuda, S., Haruki, Y. (2015) The size of retrieved lymph nodes correlates with the number of retrieved lymph nodes and is an independent prognostic 
factor in patients with stage II colon cancer. Int. J. Colorectal Dis. 30(12), 1685-93.

Ong, M.L., Schofield, J.B. (2016) Assessment of lymph node involvement in colorectal cancer. World Journal of Gastrointestinal Surgery, 8(3), 179-192.

Rollvén, E., Abraham-Nordling, M., Holm, T. et al. (2017) Assessment and diagnostic accuracy of lymph node status to predict stage III colon cancer using computed tomography. Cancer Imaging, 17, 3 .

Rotte, K.H., Kluhs, L., Kleinau, H., Kriedemann, E. (1989) Computed tomography and endosonography in the preoperative staging of rectal carcinoma. Eur. J. Radiol. 9(3), 187-190.

Sigurdson, E.R. (2003) Lymph node dissection: Is it diagnostic or therapeutic? J. Clin. Oncol. Mar. 21(6), 965-7.

Smith, N.J., Bees, N., Barbachano, Y., Norman, A.R., Swift, R.I., Brown, G. (2007) Preoperative computed tomography staging of non-metastatic colon cancer predict outcome :implication of clinical trial. British Journal of Cancer, 96, 10301036.

So, J.S., Cheong, C., Oh, S.Y., Lee, J.H., Kim, Y.B., Suh, K.W. (2017) Accuracy of preoperative local staging of primary colorectal cancer by using computed tomography: Reappraisal based on data collected at a highly organized cancer center. Annals of Coloproctology, 33(5), 192-196.

Thoeni, R.F. (1997) Colorectal cancer radiologic staging. Radiol. Clin. North Am. 35(2), 457-485.

Zagoria, R.J., Schlarb, C.A., Ott, D.J., et al. (1997) Assessment of rectal tumor infiltration utilizing endorectal MR imaging and comparison with endoscopic rectal sonography. J. Surg. Oncol. 64(4), 312-317.

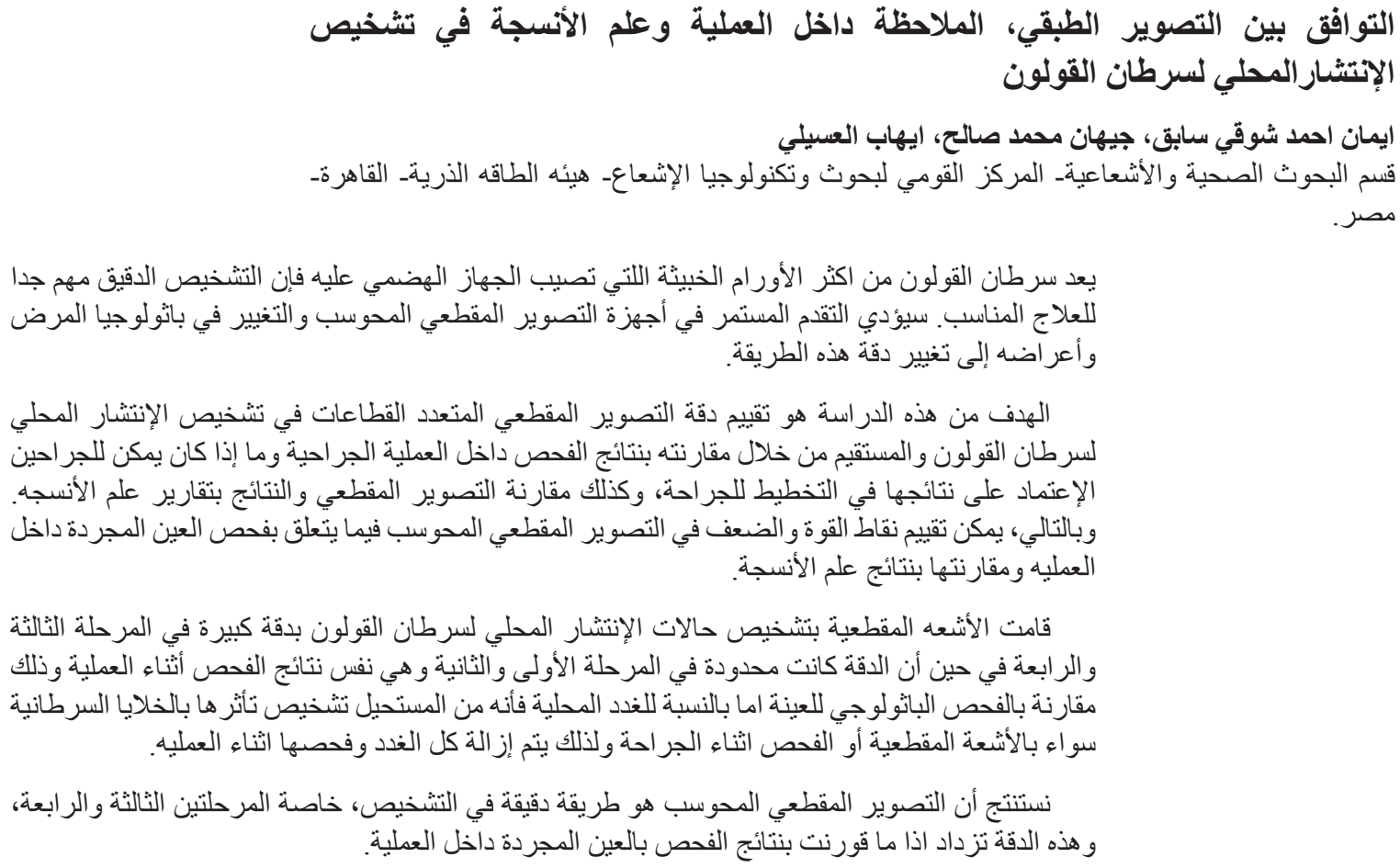

